doi:10.13108/2021-13-2-33

\title{
GENERAL SOLUTIONS OF SOME LINEAR EQUATIONS WITH VARIABLE COEFFICIENTS
}

\author{
O.V. KAPTSOV, M.M. MIRZAOKHMEDOV
}

\begin{abstract}
In this work we find general solutions to some classes of linear wave equations with variable coefficients. Such equations describe the oscillations of rods, acoustic waves, and also some models of gas dynamics are reduced to these equations.

To construct general solutions, we employ special types of Euler-Darboux transformations, namely, Levi type transformations. These transformations are first order differential substitutions. For constructing each transformation, we need to solve two linear second order ordinary differential equations. The solutions of one of these equations are determined by the solutions of the other equations by means of a differential substitution and Liouville formula. In the general case, it is not easy to solve these ordinary differential equations. However, it is possible to provide some formula for the superposition of the transformation of Levy type.

Starting with a classical wave equation with constant coefficients and employing the found transformations, we can construct infinite series of equations possessing explicit general solutions. By means of Matveev method we obtain limiting forms of iterated transformations. We provide a series of particular examples of the equations possessing general solutions.
\end{abstract}

Keywords: linear equations with variable coefficients, general solutions, limiting Levi transformations.

Mathematics Subject Classification: 35C05, 35L10, 35A09

\section{INTRODUCTION}

The propagation of one-dimensional waves in inhomogeneous media is often described by an equation of form

$$
v_{t t}=a\left(b v_{x}\right)_{x}
$$

where $a, b$ are some smooth positive functions of $x$. For instance, in the case of sound waves the function $v$ describes a pressure and $a=\rho c^{2}, b=\rho^{-1}$, where $\rho$ is a density, $c$ is the sound speed in the media [1]. If equation (1.1) models longitudinal oscillations of a rod, then $v$ describes the movements of the rod and $a=\frac{E}{\rho \omega}, b=\omega$, where $E$ is the Young modulus, $\rho$ is a density, $\omega$ is the area of the transversal cross-section of the rod. Apart of these, other models are related with equation (1.1). For instance, a system describing isentropic gas motion [4] can be reduced to form (1.1).

O.V. Kaptsov, M.M. Mirzaokhmedov, General solutions of SOME LinEar EQUations with VariABLE COEFFICIENTS.

(C) Kaptsov O.V., Mirzaokhmedov M.M. 2021.

The work is supported by Krasnoyarsk Mathematical Center financed by the Ministry of Education and Science of Russian Federation in the framework of activities for creating and developing of regional Scientific and Educational Mathematical Centers (agreement 075-02-2020-1631).

Submitted February 3, 2021. 
It is interesting to find general solutions to equations (1.1) for non-constant functions $a, b$. Some examples of such solutions can be found in [5], 3]. The general solution can be employed for solving the Cauchy problem. For constructing general solutions to some equations of form

$$
u_{x y}+a u_{x}+b u_{y}+c u=0,
$$

Laplace introduced the cascade method [2]. On the other hand, Euler [6] proposed to use differential substitutions for finding general solutions to the equations of form

$$
u_{t t}=F u_{x x}+G u_{x}+H u,
$$

where $F, G, H$ are functions on $x$. Later in the end of XIX and the beginning of XX century, Darboux [2] and other mathematicians generalized such transformations and applied them to solving geometric problems. In the last 40 years, the interest in such transformations has increased essentially in connection with the development of the theory of solitons [11], [12], 8].

In the present work we employ the Euler-Darboux transforms for finding general solutions to the equations

$$
u_{t t}=u_{x x}+g(x) u_{x}
$$

for special classes of the functions $g$. It is easy to confirm that by the point transformations, equation (1.1) is reduced to form (1.3). We find Euler-Darboux transformations mapping solutions of equation (1.2) into those of equation $(1.2)$ but with another function $g$. Starting with a function $g=0$, we can obtain infinite series of equations, by means of which we succeed to find general solutions.

Our work extend the studied initiated in [3] via using more general transformations. Moreover, we construct limiting transformations by employing the ideas of Matveev [11].

\section{EULER-DARBOUX TRANSFORMS}

As it is known [6], Euler found the conditions, under which a first order differential substitution

$$
w=M\left(u_{x}+s u\right)
$$

maps solutions of equation 1.2 into the solutions of the equation

$$
w_{t t}=F_{1} w_{x x}+G_{1} w_{x}+H_{1} w,
$$

where $M, s, F_{1}, G_{1}, H_{1}$ are functions on $x$. We provide a slightly modified formulation of this result from book [3] since it is convenient to employ it in what follows.

Lemma 2.1. Let $u$ be a solution of equation (1.2). Then the substitution

$$
w=\frac{u_{x}-(\ln h)_{x} u}{r}
$$

maps the function $u$ into a solution of equation (2.1) if

1) the function $h(x)$ solves the equation

$$
F h^{\prime \prime}+G h^{\prime}+(H+c) h=0,
$$

where $r$ is an arbitrary smooth function on $x$ and $c$ is an arbitrary constant;

2) $F_{1}, G_{1}, H_{1}$ are defined by the formulae

$$
F_{1}=F, \quad G_{1}=G+F^{\prime}+2 F(\ln r)^{\prime}, \quad H_{1}=H+\frac{\left(F r^{\prime}+G r\right)^{\prime}}{r}+F^{\prime}(\ln h)^{\prime}+2 F(\ln h)^{\prime \prime}
$$


It is easy to simplify equation (1.1) by a change of an independent variable. Indeed, let $v(t, x)=u(t, y(x))$, where $y=y(x)$ is a new variable. Calculating the derivatives $v_{t t}, v_{x}, v_{x x}$ and substituting them into (1.1), we obtain the equation

$$
u_{t t}=a b y^{\prime 2} u_{y y}+\left(a b y^{\prime \prime}+a b^{\prime} y^{\prime}\right) u_{y} .
$$

Letting $a b y^{\prime 2}=1$, we find:

$$
y=\int \frac{d x}{\sqrt{a b}} .
$$

This integral is well-defined since the functions $a, b$ are smooth and positive definite. Hence, by this change, equation (1.1) is reduced to

$$
u_{t t}=u_{x x}+G(x) u_{x} .
$$

Proposition 2.1. Substitution (2.2) maps the solutions of equation (2.5) into the solutions of the equation

$$
w_{t t}=w_{x x}+G_{1} w_{x}
$$

if the following conditions are satisfied:

1) $h(x)$ satisfies the equation

$$
h^{\prime \prime}+G h^{\prime}+c h=0, \quad c \in \mathbb{R},
$$

2)

$$
G_{1}=G+2\left(\ln \frac{h^{\prime}}{h}\right)^{\prime}
$$

3) the function $r$ satisfies the equation

$$
r^{\prime \prime}+G r^{\prime}+\left(G^{\prime}+2(\ln h)^{\prime \prime}\right) r=0 .
$$

This proposition follows from Lemma 2.1. In order to obtain 2.9 , we should let $H_{1}=H=0$ in formula (2.4).

Proposition 2.2. The substitution

$$
r=\frac{h^{\prime}}{h}
$$

maps solutions of equation (2.7) into solutions of equation (2.9). The substitution

$$
w=u-\frac{h}{h^{\prime}} u_{x}
$$

maps the solutions of equation (2.5) into the solutions of equation (2.6).

The proof is made by straightforward substitution of (2.10) into (2.9). At that, one should take into consideration equation (2.7) and its differential implications.

Remark 2.1. Substitution of type (2.11) is called Levy transform in the theory of conjugate nets [7]. We observe that knowing a general solution to equation (2.7), we do not get a general solution to equation (2.9). The general solution is found by a known Liouville formula.

Example 1. Let the function $G$ in (2.5) vanishes. Then depending on the choice of the constant $c$, we obtain three types of solutions to equation (2.7):

1) as $c=0$, the function $h$ is linear, that is,

$$
h=c_{1} x+c_{2}, \quad c_{1}, c_{2} \in \mathbb{R} ;
$$

2) as $c=-k^{2}<0$, the solution reads as

$$
h=c_{1} \exp (k x)+c_{2} \exp (-k x), \quad c_{1}, c_{2} \in \mathbb{R} ;
$$


3) as $c=k^{2}>0$, the function $h$ is

$$
h=c_{1} \sin (k x)+c_{2} \cos (k x) .
$$

Let us consider the first option. In this case the general solution of equation 2.9 reads as

$$
r=\frac{d_{1}}{x+b}+d_{2}(x+b)^{2}, \quad d_{1}, d_{2} \in R, \quad b=\frac{c_{2}}{c_{1}} .
$$

Hence, according to (2.8), we obtain

$$
G_{1}=\frac{4 m(x+b)^{3}-2}{(x+b)\left(m(x+b)^{3}+1\right)}, \quad m=\frac{d_{2}}{d_{1}} .
$$

Therefore, in accordance with Proposition 2.1, a general solution to equation (2.6) in this case reads as

$$
w=\frac{(x+b) u_{x}+u}{d_{1}+d_{2}(x+b)^{3}},
$$

where $u=X(x+t)+T(x-t)$, and $X, T$ are arbitrary smooth function.

Let us briefly dwell on the second option. In this case two solutions of equation $(2.9)$ are of the form

$$
r_{1}=k \frac{\exp (k x)-b \exp (-k x)}{\exp (k x)+b \exp (-k x)}, \quad r_{2}=\frac{k x(\exp (2 k x)-b)-2 b}{\exp (2 k x)+b}, \quad b=\frac{c_{2}}{c_{1}} .
$$

The function $G_{1}$ is found by formula (2.8) and as $r=r_{1}$, it reads as

$$
G_{1}=\frac{8 b k \exp (2 k x)}{b^{2} \exp (4 k x)-1}
$$

The general solution of equation (2.6) with this function $G_{1}$ is found by means of substitution (2.2) and it reads as

$$
w=X+T-\frac{\left(X^{\prime}+T^{\prime}\right)(\exp (k x)+b \exp (-k x))}{k(\exp (k x)-b \exp (-k x))},
$$

where $X(x+t), T(x-t)$ are arbitrary smooth function and the prime stands for the derivative. It is obvious that as $b<0$, solution $(2.12)$ is smooth. The third option can be considered in the same way.

In [3], a lemma was proved on a sequence of transforms of Levy type.

Lemma 2.2. Let $h_{1}, \ldots, h_{n}$ be linearly independent solutions of equation (2.7) corresponding to parameters $c_{1}, \ldots, c_{n}$ and $c_{i} \neq c_{j}$ as $i \neq j$. Then the transform

$$
z=\frac{W\left(u, h_{1}, \ldots, h_{n}\right)}{W\left(h_{1}^{\prime}, \ldots, h_{n}^{\prime}\right)}
$$

where $W$ is the Wronskian, maps the solutions of equation 2.5 into the solutions of equation

$$
z_{t t}=z_{x x}+G_{n} z_{x}
$$

and the function $G_{n}$ is defined by the formula

$$
G_{n}=G+2 \frac{d}{d x}\left(\ln \frac{W\left(h_{1}^{\prime}, \ldots, h_{n}^{\prime}\right)}{W\left(h_{1}, \ldots, h_{n}\right)}\right) .
$$

In [3], one can find examples concerning this lemma. In work [9], there was studied a Levy sequence appearing in the theory of conjugate nets. 


\section{LIMITING TRANSFORMATIONS AND EXAMPLES}

It was assumed in Lemma 2.2 on sequence of Levy type transformations that the functions $h_{i}$ correspond to mutually different parameters $c_{i}$. Here we consider the case of coinciding parameters. Below we shall sometimes employ notations $\partial_{x}, \partial_{k}$ for the operators of differentiation with respect to $x$ and $k$, respectively. We shall also employ standard notations for the derivatives.

Lemma 3.1. Let $h(x, k)$ be a solution of the equation

$$
h^{\prime \prime}+g h^{\prime}-k^{2} h=0,
$$

where $g$ is a smooth function on $x, k \in \mathbb{R}$. Then the transformation

$$
z=\frac{W\left(u, h, \partial_{k} h, \ldots, \partial_{k}^{m} h\right)}{W\left(\partial_{x} h, \partial_{k} \partial_{x} h, \ldots, \partial_{k}^{m} \partial_{x} h\right)}
$$

maps the solutions of the equation

$$
u_{t t}=u_{x x}+g u_{x}
$$

into the solutions of the equation

$$
z_{t t}=z_{x x}+g_{m+1} z_{x}
$$

where the function $g_{m+1}$ is given by the formula

$$
g_{m+1}=g+2 \partial_{x}\left(\ln \frac{W\left(\partial_{x} h, \partial_{k} \partial_{x} h, \ldots, \partial_{k}^{m} \partial_{x} h\right)}{W\left(h, \partial_{k} h, \ldots, \partial_{k}^{m} h\right)}\right) .
$$

Proof. In our arguging, we shall employ the ideas of work by Matveev [10]. For simplicity, we consider the case $m=1$. Let $h(x, k)$ be a solution of equation (3.1). We introduce the notations $h^{1}=h\left(x, k_{1}\right), h^{2}=h\left(x, k_{2}\right)$. According Taylor formula, we have

$$
h^{2}=h^{1}+\varepsilon \partial_{k} h^{1}+o(\varepsilon),
$$

where $\varepsilon=k_{2}-k_{1}$ and $o(\varepsilon)$ is the Landau symbol.

We are going to pass to the limit $\varepsilon \rightarrow 0$ in formulae (2.13), (2.15). The Wronskian $W\left(u, h^{1}, h^{2}\right)$ is written as follows:

$$
\begin{aligned}
W\left(u, h^{1}, h^{2}\right) & =\left|\begin{array}{ccc}
u & h^{1} & h^{1}+\varepsilon \partial_{k} h^{1}+o(\varepsilon) \\
u_{x} & h_{x}^{1} & h_{x}^{1}+\varepsilon \partial_{k} h_{x}^{1}+o_{x}(\varepsilon) \\
u_{x x} & h_{x x}^{1} & h_{x x}^{1}+\varepsilon \partial_{k} h_{x x}^{1}+o_{x x}(\varepsilon)
\end{array}\right|=\varepsilon\left|\begin{array}{ccc}
u & h^{1} & \partial_{k} h^{1}+o \\
u_{x} & h_{x}^{1} & \partial_{k} h_{x}^{1}+o_{x} \\
u_{x x} & h_{x x}^{1} & \partial_{k} h_{x x}^{1}+o_{x x}
\end{array}\right| \\
& =\varepsilon W\left(u, h^{1}, \partial_{k} h^{1}+o(\varepsilon)\right) .
\end{aligned}
$$

In the same way we obtain the formula

$$
W\left(h_{x}^{1}, h_{x}^{2}\right)=\varepsilon W\left(\partial_{x} h^{1}, \partial_{k} \partial_{x} h^{1}+o(\varepsilon)\right) .
$$

Hence, we have

$$
\lim _{\varepsilon \rightarrow 0} \frac{W\left(u, h^{1}, h^{2}\right)}{W\left(h_{x}^{1}, h_{x}^{2}\right)}=\frac{W\left(u, h^{1}, \partial_{k} h^{1}\right)}{W\left(\partial_{x} h^{1}, \partial_{k} \partial_{x} h^{1}\right)} .
$$

In the same way, by formula (2.15) we obtain expression (3.5). The validity of the lemma as $m=1$ can be also confirmed straightforwardly. The proof is complete.

Remark 3.1. We can also obtain a more general statement than Lemma 3.1, as a similar statement in [10]. It corresponds to the case when not all constants $c_{i}$ in Lemma 2.2 coincide. The arguing are generally the same but the formulae become more bulky. 
Example 2. Let $g=0, k \neq 0, m=1$. Then a solution of equation (3.1) reads as

$$
h=a_{1} \sinh (k x)+a_{2} \cosh (k x) .
$$

To simplify the formulae we assume that $a_{2}=0$. Then, according (3.5), the function $g_{2}$ is

$$
g_{2}=-\frac{2 k\left(4 k x \cosh ^{2}(k x)-\sinh (2 k x)-2 k x\right)}{\cosh ^{2}(k x) \sinh ^{2}(k x)-k^{2} x^{2}} .
$$

The general solution of equation (3.4) reads as

$$
z=\frac{(\cosh (k x) \sinh (k x)-k x) g^{\prime \prime}+\left(-2 k \cosh ^{2}(k x)+2 k\right) g^{\prime}+\cdots+k\left(k^{2} x f-x f^{\prime \prime}+2 f^{\prime}\right)}{k^{2}(\cosh (k x) \sinh (k x)+k x)} .
$$

Here $f(x+t), g(x-t)$ are arbitrary smooth functions. In formula (3.7) and in solution (3.8) we can pass to the limit as $k \rightarrow 0$. Then

$$
g_{2} \underset{k \rightarrow 0}{\longrightarrow} \tilde{g_{2}}=-\frac{4}{x}, \quad z \underset{k \rightarrow 0}{\longrightarrow} \tilde{z}=\frac{1}{3}\left(f^{\prime \prime}+g^{\prime \prime}\right) x^{2}-\left(f^{\prime}+g^{\prime}\right) x+f+g .
$$

Let $m=2$. In this case the expressions for $g_{3}$ and $z$ are rather cumbersome but they can be easily found by means of computer algebra. However, if we pass to the limit as $k \rightarrow 0$ in the formulae for $g_{3}$ and $z$, then we obtain:

$$
g_{3}=-\frac{6}{x}, \quad z=-\frac{1}{15}\left(f^{\prime \prime \prime}+g^{\prime \prime \prime}\right) x^{3}+\frac{2}{5}\left(f^{\prime \prime}+g^{\prime \prime}\right) x^{2}-\left(f^{\prime}+g^{\prime}\right) x+f+g .
$$

It is also possible to make the inverse passing from equation (1.3) to equation (1.1) by transforming the independent variable. Indeed, let $u(t, x)=v(t, y(x))$. Then we substitute the derivatives

$$
u_{t t}=v_{t t}, \quad u_{x}=y^{\prime} v_{y}, \quad u_{x x}=y^{\prime 2} v_{y y}+y^{\prime \prime} v_{y}
$$

into 1.3 and we obtain

$$
v_{t t}=y^{\prime 2} v_{y y}+\left(y^{\prime \prime}+g y^{\prime}\right) v_{y}
$$

Comparing with (1.1), we arrive at the system

$$
a b=y^{\prime 2}, \quad a b_{y}=y^{\prime \prime}+g y^{\prime},
$$

where $a, b$ are functions on $y$ and $g$ is a function of $x$. Expressing $a$ from the first equation in (3.9) and substituting it into the second equation, we obtain:

$$
\frac{b_{y}}{b}=\frac{y^{\prime \prime}+g y^{\prime}}{y^{\prime 2}} .
$$

If we choose functions $y(x), g(x)$ and then express the right hand side of (3.10) via $y$, we obtain a linear second order differential equation for the function $b$. Solving it, we find $b$ and then $a(y)$.

Example 3. Let $g=-\frac{2}{x}, y=\operatorname{coth}(x)$. Then the equation for the function $b$

$$
b_{y}=\frac{2\left(2 \operatorname{coth}^{2}(y)-1\right)}{\operatorname{coth}(y)} b
$$

has a solution

$$
b=A \frac{\sinh ^{4}(y)}{\cosh ^{2}(y)}, \quad A \in \mathbb{R} .
$$

Therefore, the function $a$ is of the form

$$
a=\frac{\cosh ^{2}(y)}{A}
$$


As it has been mentioned above, the general solution of the equation

$$
u_{t t}=u_{x x}-\frac{2}{x} u_{x}
$$

is written in the form

$$
u=x V_{x}-V,
$$

where $V=f_{1}(x+t)+f_{2}(x-t)$ and $f_{1}, f_{2}$ are arbitrary smooth functions. Therefore, a general solution of the equation

$$
v_{t t}=\cosh ^{2}(y)\left(\frac{\sinh ^{4}(y)}{\cosh ^{2}(y)} v_{y}\right)_{y}
$$

is of the form

$$
v=\operatorname{coth}(y)\left(f_{1}^{\prime}(\operatorname{coth}(y)+t)+f_{2}^{\prime}(\operatorname{coth}(y)-t)\right)-\left(f_{1}(\operatorname{coth}(y)+t)+f_{2}(\operatorname{coth}(y)-t)\right) .
$$

Example 4. Let $g=\frac{2}{x}, y=\operatorname{coth}(x)$. Then the equation for the function $b$

$$
b_{y}=\frac{2 b}{\operatorname{coth}(y)}
$$

has a solution

$$
b=\frac{\cosh ^{2}(y)}{A}, \quad A \in \mathbb{R} .
$$

Therefore, the function $a$ reads as

$$
a=A \frac{\sinh ^{4}(y)}{\cosh ^{2}(y)} .
$$

As it has been mentioned above, the general solution of the equation

$$
u_{t t}=u_{x x}+\frac{2}{x} u_{x}
$$

is written in the form

$$
u=\frac{f_{1}(x+t)+f_{2}(x-t)}{x},
$$

where $f_{1}, f_{2}$ are arbitrary smooth functions. Therefore, the general solution of the equation

$$
v_{t t}=\frac{\sinh ^{4}(y)}{\cosh ^{2}(y)}\left(\cosh ^{2}(y) v_{y}\right)_{y}
$$

reads as

$$
v=\frac{f_{1}(\operatorname{coth}(y)+t)+f_{2}(\operatorname{coth}(y)-t)}{\operatorname{coth}](y)} .
$$

Example 5. Let $g=\frac{2}{\sinh (x)}, y=\exp (x)$. Then the function $b$ reads as

$$
b=A \frac{y(y-1)^{2}}{(y+1)^{2}}
$$

where $A$ is an arbitrary constant. In this case the function $a$ is given by the formula

$$
a=\frac{y(y+1)^{2}}{A(y-1)^{2}} .
$$

The general solution (1.3) with this function $g$ is given by formula (2.12). Substituting $x=\ln y$ and the found values of $a, b$, we obtain a general solution of equation (1.1) with functions (3.11), 3.12 . 


\section{BIBLIOGRAPHY}

1. L.M. Brekhovskikh. Waves in layered media. Nauka, Moscow (1973). [Academic Press, New York (1980).]

2. G. Darboux. Lectures on general theory of surfaces. V. 2. Inst. Comp. Stud., Moscow (2013). [Leçons sur la théorie générale des surfaces et les applications géométriques du calcul infinitésimal. Teil 1 u. 2. Gauthier-Villars, Paris. (1887, 1889).]

3. O.V. Kaptsov. Methods for integrating partial differential equations. Fizmatlit, Moscow (2009). (in Russian).

4. L.V. Ovsyannikov. Lectures on the fundamentals of gas dynamics. Nauka, Moscow (1981).

5. A.D. Polyanin, V.F. Zaitsev. Handbook of nonlinear partial differential equations. Fizmatlit, Moscow (2002). [CRC Press, Boca Raton, FL (2011).]

6. L. Euler. Integral calculus. V. 3. GIFML, Moscow (1958). [Principles of integral calculus. Part III. B.G. Teubner, Leipzig (1914).]

7. L. Eisenhart. Transformations of surfaces. Chelsea Publ., Princeton (1962).

8. C. Gu, H. Hu, Z. Zhou. Darboux Transformations in Integrable Systems. Theory and their Applications to Geometry. Springer, New York (2005).

9. E.S. Hammond. Periodic conjugate nets // Ann. Math. 22:4, 238-261 (1921).

10. V.B. Matveev. Generalized Wronskian formula for solutions of the KdV equations: first applications // Phys. Lett. A. 166:3, 205-208 (1992).

11. V.B. Matveev, M.A. Salle. Darboux transformations and solitons. Springer-Verlag, New York (1991).

12. C. Rogers, W.K. Schief. Backlund and Darboux Transformations Geometry and Modern Applications in Soliton Theory. Cambridge University Press, Cambridge (2002).

Oleg Viktorovich Kaptsov,

Institute of Computational Modeling SB RAS,

Akademgorodok, 50/44,

660036, Krasnoyarsk, Russia

E-mail: kaptsov@icm.krasn.ru

Mansur Mavlonovich Mirzaokhmedov,

Siberian Federal University,

Svobodny av. 79,

660041, Krasnoyarsk, Russia

E-mail: mansur.mirzoahmedov@mail.ru 